\title{
Estado nutricional e fatores associados em escolares domiciliados na área rural e urbana
}

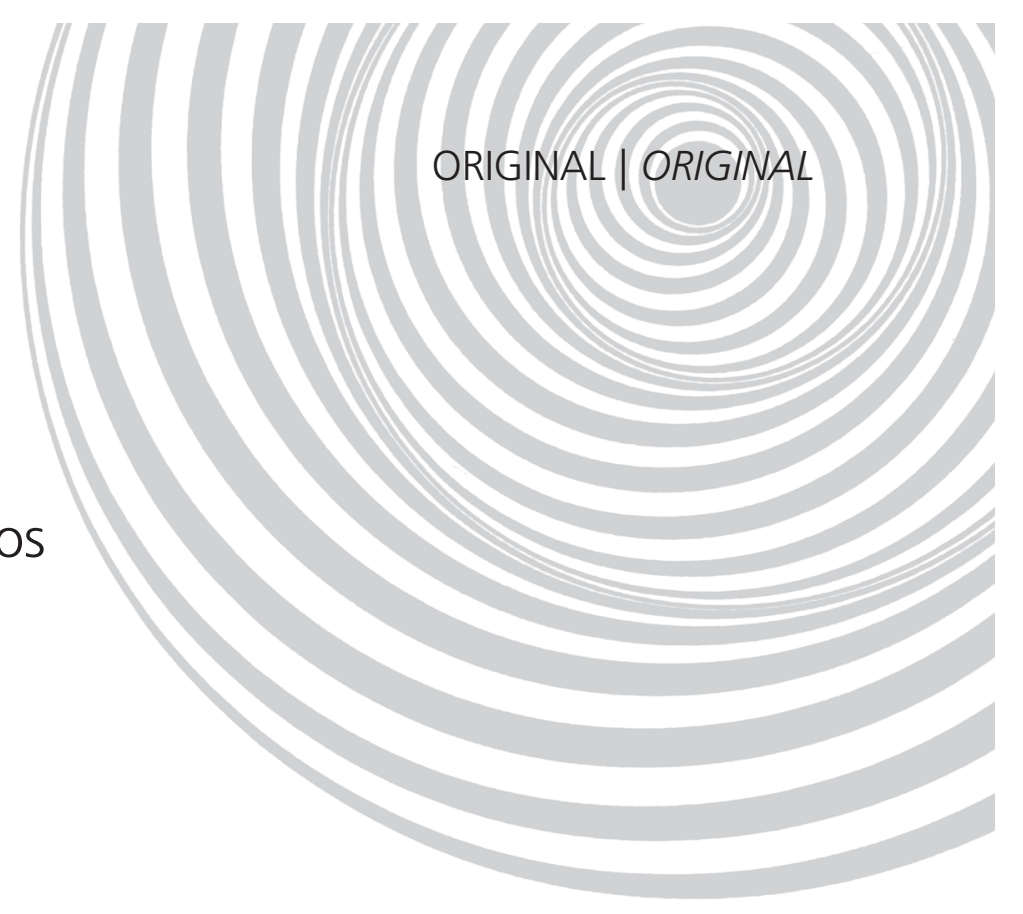

\author{
Nutritional status and associated \\ factors in schoolchildren living \\ in rural and urban areas
}

\author{
Andreia PELEGRINI' \\ Diego Augusto Santos SILVA' \\ Edio Luiz PETROSKI ${ }^{1}$ \\ Maria Fátima GLANER²
}

RE S U M O

\section{Objetivo}

Verificar o estado nutricional de escolares domiciliados nas áreas urbana e rural da Região Sul do Brasil e analisar sua associação com fatores demográficos e nível de atividade física.

\section{Métodos}

Participaram do estudo 1415 escolares (720 rapazes e 695 moças), sendo 878 da área urbana e 537 da área rural. Foram pesquisadas informações demográficas (sexo, idade, área de domicílio), antropométricas (massa corporal, estatura) e do nível de atividade física. O estado nutricional - desnutrição e excesso de peso - foi determinado pelo índice de massa corporal a partir dos critérios propostos pela International Obesity Task Force. O nível de atividade física foi classificado em duas categorias: mais ativo ou menos ativo.

\section{Resultados}

A prevalência de desnutrição foi de 11,4\% (IC95\%=9,85-13,16) e excesso de peso de 11,2\% (IC95\%=9,66-12,95). A prevalência de excesso de peso foi superior nos escolares domiciliados na área urbana $(14,0 \%$; IC $95 \%=12,29-15,91)$ em relação aos da área rural $(6,7 \%$; IC $95 \%=5,51-8,12)$. Foi verificado, nos rapazes, que os menos ativos fisicamente $(O R=1,74 ; I C 95 \%=1,03-2,94)$ apresentaram chance maior de ter desnutrição. Além disso, os adolescentes da área urbana ( $O R=3,40$; IC $95 \%=1,88-6,17)$ e os menos ativos fisicamente $(\mathrm{OR}=1,88$; IC95\%=1,07-3,33) apresentaram maiores chances de excesso de peso. As moças de 10 a 13 anos apresentaram maior chance de desnutrição $(O R=1,95$; IC95\%=1,17-3,24) e aquelas residentes na área urbana $(\mathrm{OR}=1,75$; IC $95 \%=1,03-2,99)$, mais chance de excesso de peso.

\footnotetext{
1 Universidade Federal de Santa Catarina, Programa de Pós-Graduação em Educação Física, Núcleo de Pesquisa em Cineantropometria e Desempenho Humano. Caixa Postal 476, Campus Universitário, Trindade, 88040-900, Florianópolis, SC, Brasil. Correspondência para/Correspondence to: A. PELEGRINI. E-mail: <petroski@cds.ufsc.br>.

2 Universidade Católica de Brasília, Programa de Pós-Graduação em Educação Física, Grupo de Estudos em Medida e Avaliação, Cineantropometria e Desempenho Humano. Brasília, DF, Brasil.
} 
840 A. PELEGRINI et al.

\section{Conclusão}

A prevalência de desnutrição encontrada ainda é elevada em escolares. O excesso de peso assemelha-se ao que tem sido observado nas regiões Sul e Sudeste do Brasil. Rapazes com baixo nível de atividade física e moças de 10 a 13 anos apresentam maior exposição à desnutrição. Moças e rapazes domiciliados na área urbana e rapazes com baixo nível de atividade física apresentam maior exposição aos riscos decorrentes do excesso de peso corporal.

Termos de indexação: Estado nutricional. Obesidade. Saúde do adolescente. Sobrepeso.

\section{A B S T R A C T}

\section{Objective}

The objectives of this study were to assess the nutritional status of schoolchildren living in urban and rural areas in the southern region of Brazil, and investigate a possible association between nutritional status and demographic factors and level of physical activity.

\section{Methods}

A total of 1,415 schoolchildren (720 boys and 695 girls) participated in the study; of these, 878 lived in rural areas and 537 lived in urban areas. Demographic (gender, age, residence location) and anthropometric (body weight and height) data were collected and level of physical activity investigated. Nutritional status (malnutrition and excess weight) was classified according to body mass index following the International Obesity Task Force criteria. The level of physical activity was classified into two categories (more or less active).

\section{Results}

The prevalence of malnutrition was $11.4 \%(C / 95 \%=9.85-13.16)$ and the prevalence of excess weight was $11.2 \%(C / 95 \%=9.66-12.95)$. The prevalence of excess weight was higher among adolescents living in urban areas (14.0\%; C195\%=12.29-15.91) than among those living in rural areas (6.7\%; C195\%=5.51-8.12). Boys with lower levels of physical activity $(O R=1.74 ; C 195 \%=1.03-2.94)$ were more likely to be malnourished. On the other hand, adolescents living in urban areas (OR=3.40; $C 195 \%=1.88-6.17)$ and less physically active $(O R=1.88$; $C 195 \%=1.07-3.33)$ were more likely to be overweight or obese. Girls aged 10 to 13 years were more likely to be malnourished $(O R=1.95$; $C 195 \%=1.17-3.24)$ and those living in urban areas $(O R=1.75$; C195\%=1.03-2.99) were more likely to be overweight or obese.

\section{Conclusion}

The prevalence of malnourished schoolchildren is still high. In contrast, the prevalence of excess weight was similar to that observed in South and Southeast Brazil. Boys with low levels of physical activity and girls aged 10 to 13 years are more exposed to malnutrition. Boys and girls living in urban areas and boys with low levels of physical activity are more exposed to the risk of becoming overweight or obese.

Indexing terms: Nutritional status. Obesity. Adolescent health. Overweight.

\section{N T R O D U Ç Ã O}

A presença da desnutrição, deficiência de micronutrientes, excesso de peso e outras doenças não transmissíveis coexistentes nas mesmas comunidades e, muitas vezes, no mesmo domicílio caracterizam a transição nutricional. No Brasil, ao mesmo tempo em que se observa uma redução contínua dos casos de desnutrição ${ }^{1}$, são observadas prevalências crescentes de excesso de peso nas formas de sobrepeso e obesidade ${ }^{2}$. Nos países desenvolvidos e em desenvolvimento, a ascensão do excesso de peso tem sido observada principalmente entre os adolescentes e adultos jovens. Esse fato é bastante preocupante, pois a obesidade nessa fase da vida é fator de risco para a obesidade na idade adulta ${ }^{3}$. Atrelados a esse contexto, o sobrepeso e a obesidade estão associados a doenças crônicas não-transmissíveis, tais como: hipertensão arterial $^{4,5}$, dislipidemia ${ }^{5}$, diabetes mellitus tipo $2^{4}$ e outros fatores de risco para doença arterial coronariana ${ }^{6}$. Além disso, tem sido constatada associação com níveis elevados de ansiedade $^{7}$ e redução no desempenho escolar ${ }^{8}$. 
A World Health Organization ${ }^{9}$ tem estimado que, a cada ano, 1,9 e 2,6 milhões de pessoas morrem como resultado da inatividade física e do sobrepeso/obesidade, respectivamente. Dessa forma, supõe-se que o excesso de peso apresente forte relação com o estilo de vida sedentário ${ }^{10}$, além disso, a inatividade física na adolescência é um forte indicador do risco de obesidade na idade adulta, favorecendo um círculo vicioso entre obesidade e sedentarismo ${ }^{11}$.

Algumas pesquisas internacionais foram realizadas para verificar o estado nutricional em adolescentes das áreas urbana e rural12,13. Em contrapartida, no Brasil, estudos envolvendo adolescentes da área rural são escassos e, portanto, necessários. Além disso, o estado nutricional de crianças e adolescentes pode variar de uma área para outra ${ }^{14}$, justificando a realização desse trabalho. Dessa forma, o presente estudo tem como objetivos: a) verificar o estado nutricional em escolares domiciliados na área urbana e rural da região Sul do Brasil e b) analisar a associação entre o estado nutricional e os fatores demográficos e nível de atividade física.

\section{MÉ T O D O S}

Trata-se de um estudo epidemiológico transversal, de base escolar, realizado com escolares de 10 a 17 anos, domiciliados nos meios urbano e rural, matriculados em escolas públicas. A pesquisa foi aprovada pelo Comitê de Ética em Pesquisa com Seres Humanos da Universidade Federal de Santa Catarina, protocolo n ${ }^{\circ} 217$, em 29 de setembro de 2008 . Todos os participantes assinaram um termo de consentimento livre e esclarecido antes da coleta de dados.

O estudo foi conduzido em escolares das áreas urbana e rural de quatro municípios: três do estado de Santa Catarina - Chapecó, Concórdia e Saudades - e um do estado do Rio Grande do Sul - Erval Grande. Pelo fato de o município de Chapecó apresentar população urbana $(13,2 \%)$ superior à média da Região Sul do Brasil, a amostra urbana foi composta por adolescentes domiciliados nessa cidade. A amostra rural foi constituída por adolescentes domiciliados nas áreas rurais dos municípios de Concórdia (SC), Saudades (SC) e Erval Grande (RS) pelo fato de possuírem uma população rural sensivelmente superior $-62,6 \%, 65 \%$ e $28,3 \%$ respectivamente - à população rural da Região Sul do país $(21,6 \%)^{14}$.

As escolas das redes municipal, estadual e federal foram englobadas no presente estudo na tentativa de se obter uma amostragem representativa de escolares dos dois domicílios. Isso foi necessário, pois: a) a maioria das escolas do meio rural é municipalizada e atende somente o ensino fundamental; b) parte das escolas agrotécnicas federais localiza-se no interior e a maioria dos seus alunos é procedente de áreas rurais; c) a maioria das escolas que possuem ensino médio é estadual e localiza-se na área urbana. As três escolas existentes em Erval Grande e Saudades que atendem alunos rurais, da faixa etária abrangida no estudo, foram envolvidas. De Concórdia foi escolhida a Escola Agrotécnica Federal para compor a amostra nas faixas etárias mais elevadas. Todos os alunos rurais foram convidados a participar. A adesão foi superior a $95 \%$ dos que estavam presentes na aula de educação física - dia da coleta. Para selecionar os escolares urbanos, foi sorteada uma escola de cada região da cidade de Chapecó, totalizando cinco escolas. Em cada uma foram sorteadas turmas para atingir o número de escolares determinado para cada faixa etária. Posteriormente, os alunos foram convidados a participar do estudo. Dos que estavam presentes no momento da coleta, aproximadamente $90 \%$ voluntariaram-se. Participaram do estudo 1420 adolescentes. Foram considerados como perda amostral $(n=5)$ os escolares que não tiveram sua estatura mensurada. A amostra final foi composta de 1415 adolescentes, sendo 878 domiciliados na área urbana e 537 na área rural.

Foram coletadas informações demográficas (sexo, idade e área domiciliar), antropométricas (massa corporal e estatura) e sobre o Nível de Atividade Física (NAF). A massa corporal 
e a estatura foram mensuradas seguindo procedimentos padronizados ${ }^{15}$. O estado nutricional foi verificado por meio do Índice de Massa Corporal (IMC), assim calculado: IMC = massa corporal/estatura². Para a classificação do estado nutricional, foram utilizados pontos de corte amplamente aceitos na literatura: desnutrição ${ }^{16}$ : $I M C<18,5 \mathrm{~kg} / \mathrm{m}^{2}$, eutrófico ${ }^{17}:$ IMC entre 18,5 e $25 \mathrm{~kg} / \mathrm{m}^{2}$; excesso de peso: IMC $>25 \mathrm{~kg} / \mathrm{m}^{2}$, por sexo e idade. Adotou-se a expressão excesso de peso tanto para se referir ao sobrepeso quanto à obesidade. O NAF foi estimado pelo questionário desenvolvido por Pate ${ }^{18}$, traduzido e adaptado por Nahas $^{19}$. Os próprios adolescentes responderam ao questionário, em sala de aula, com a orientação de um dos pesquisadores envolvidos no estudo. Esse instrumento considera as atividades ocupacionais diárias e de lazer para classificar o indivíduo em um dos quatro níveis: inativo, moderadamente ativo, ativo e muito ativo. Essa variável foi dicotomizada em mais ativo (ativo e muito ativo) e menos ativo fisicamente (pouco ativo e inativo).

Inicialmente, foi realizada a descrição da amostra por sexo. Nas comparações entre duas proporções, foi aplicado o teste de significância para diferenças entre as proporções. Valores categóricos foram comparados por meio do teste qui-quadrado, e para os valores contínuos utilizou-se o teste $t$ de Student para amostras independentes. Como a variável dependente (estado nutricional) foi composta por três categorias (eutró- fico, desnutrição e excesso de peso), empregou-se a técnica de regressão logística multinomial para estimar a associação entre o estado nutricional e as variáveis independentes (idade, área de domicílio e NAF). A categoria eutrófico foi adotada como o grupo de referência. Em todas as análises, as variáveis foram trabalhadas de forma dicotômica: idade: 10 a 13 anos e 14 a 17 anos; área de domicílio: urbana e rural; NAF: mais ativo ou menos ativo fisicamente. Em todas as análises adotou-se nível de significância de 5\% ( $p<0,05$ ou IC95\%).

\section{RESULTADOS}

Dos 1415 escolares investigados, 50,9\% (720) são rapazes e 49,1\% (695), moças. Com relação aos valores descritivos, foram observadas diferenças entre os sexos, na massa corporal e na estatura $(p<0,05)$, com valores superiores para os rapazes (massa corporal $=$ Média $(M)=49$,8 Desvio-Padrão $(D P)=13,0 \mathrm{~kg}$ estatura $=M=160,0$ $D P=12,2 \mathrm{~cm}$ ) em relação às moças (massa corpo$\mathrm{ral}=M=48,4, D P=10,3 \mathrm{~kg}$; estatura $=M=154,0$, $\mathrm{DP}=8,2 \mathrm{~cm})$.

Prevalências maiores de excesso de peso são verificadas entre os adolescentes de 10 a 13 anos e naqueles domiciliados na área urbana $(p<0,05)$ (Tabela 1).

Tabela 1. Estado nutricional em adolescentes de acordo com a faixa etária, área de domicílio e nível de atividade física. Chapecó, Concórdia, Saudades (SC) e Erval Grande (RS), 2000.

\begin{tabular}{|c|c|c|c|c|c|c|c|c|c|}
\hline \multirow{2}{*}{ Variáveis } & \multicolumn{2}{|c|}{ Eutrofia } & \multirow{2}{*}{$p$} & \multicolumn{2}{|c|}{ Desnutrição } & \multirow{2}{*}{$p$} & \multicolumn{2}{|c|}{ Excesso de peso } & \multirow{2}{*}{$p$} \\
\hline & $n$ & $\%$ & & $n$ & $\%$ & & $n$ & $\%$ & \\
\hline \multicolumn{10}{|c|}{ Faixa etária (anos) } \\
\hline $10-13$ & 468 & 74,9 & \multirow{2}{*}{0,008} & 74 & 11,8 & \multirow{2}{*}{0,599} & 83 & 13,3 & \multirow{2}{*}{0,002} \\
\hline $14-17$ & 626 & 79,2 & & 88 & 11,1 & & 76 & 9,6 & \\
\hline \multicolumn{10}{|c|}{ Área de domicílio } \\
\hline Urbana & 652 & 74,3 & \multirow{2}{*}{$<0,001$} & 103 & 11,7 & \multirow{2}{*}{0,598} & 123 & 14,0 & \multirow{2}{*}{$<0,001$} \\
\hline Rural & 442 & 82,3 & & 59 & 11,0 & & 36 & 6,7 & \\
\hline \multicolumn{10}{|l|}{$N A F$} \\
\hline Menos ativo & 330 & 75,7 & \multirow{2}{*}{0,180} & 51 & 11,7 & \multirow{2}{*}{0,848} & 55 & 12,6 & \multirow{2}{*}{0,129} \\
\hline Mais ativo & 755 & 77,9 & & 110 & 11,4 & & 104 & 10,7 & \\
\hline
\end{tabular}

n: valor absoluto; (\%): valor relativo; $p$ : nível de significância; NAF: nível de atividade física. 
A prevalência e as razões de chance de desnutrição e excesso de peso para o sexo masculino estão apresentadas na Tabela 2. A desnutrição foi significativamente $(p<0,05)$ associada com o NAF, indicando que os adolescentes menos ativos fisicamente apresentam $74 \%$ mais chances de ter o desfecho quando comparados com os mais ativos fisicamente. O excesso de peso foi significativamente $(p<0,05)$ associado com a área de domicílio e com o NAF, e os adolescentes domiciliados na área urbana e os menos ativos fisica- mente apresentam maiores riscos de excesso de peso (Tabela 2).

A desnutrição foi significativamente $(p<0,05)$ associada com a idade: as escolares de 10 a 13 anos apresentaram duas vezes mais chance de desnutrição que as escolares de 14 a 17 anos. 0 excesso de peso foi significativamente $(p<0,05)$ associado com a área de domicílio. Esses achados revelam que as escolares que residem na área urbana apresentam $75 \%$ mais chance de excesso de peso que aquelas da área rural (Tabela 3).

Tabela 2. Associação da desnutrição e do excesso de peso com fatores associados na análise multinomial para o sexo masculino (Categoria de referência: IMC: entre 18,5 kg/m² e 25 kg/m²). Chapecó, Concórdia, Saudades (SC) e Erval Grande (RS), 2000.

\begin{tabular}{|c|c|c|c|c|c|}
\hline & \multicolumn{2}{|c|}{ Eutrofia } & \multicolumn{2}{|c|}{ Desnutrição } & \multirow{2}{*}{$\frac{\text { Excesso de peso }}{\text { OR (IC95\%) }}$} \\
\hline & $n$ & $\%$ & OR (IC95\%) & $\%$ & \\
\hline \multicolumn{6}{|c|}{ Faixa etária (anos) } \\
\hline $10-13$ & 234 & 10,3 & $0,74(0,46-1,18)$ & 14,2 & $1,61(1,00-2,60)$ \\
\hline $14-17$ & 316 & 14,1 & 1,00 & 8,8 & 1,00 \\
\hline \multicolumn{6}{|c|}{ Área de domicílio } \\
\hline Urbana & 313 & 12,7 & $1,24(0,78-1,98)$ & 15,0 & $3,40(1,88-6,17)^{*}$ \\
\hline Rural & 237 & 12,2 & 1,00 & 5,2 & 1,00 \\
\hline \multicolumn{6}{|l|}{ NAF } \\
\hline Menos ativo & 96 & 17,1 & $1,74(1,03-2,94)^{*}$ & 14,3 & $1,88(1,07-3,33)^{*}$ \\
\hline Mais ativo & 448 & 11,5 & 1,00 & 10,5 & 1,00 \\
\hline
\end{tabular}

${ }^{*} p<0,05$.

OR: odds ratio; IC95\%: intervalo de confiança; NAF: nível de atividade física.

Tabela 3. Associação entre desnutrição e excesso de peso e fatores associados na análise multinomial para o sexo feminino (Categoria de referência: IMC: entre 18,5kg/m² e 25 kg/m²). Chapecó, Concórdia, Saudades (SC) e Erval Grande (RS), 2000.

\begin{tabular}{|c|c|c|c|c|c|}
\hline & \multicolumn{2}{|c|}{ Eutrofia } & \multicolumn{2}{|c|}{ Desnutrição } & \multirow{2}{*}{$\begin{array}{c}\text { Excesso de peso } \\
\text { OR (IC95\%) }\end{array}$} \\
\hline & $\mathrm{n}$ & $\%$ & OR (IC95\%) & $\%$ & \\
\hline \multicolumn{6}{|c|}{ Faixa etária (anos) } \\
\hline $10-13$ & 234 & 13,3 & $1,95(1,17-3,24)^{*}$ & 12,4 & $1,38(0,86-2,23)$ \\
\hline $14-17$ & 310 & 7,9 & 1,00 & 10,5 & 1,00 \\
\hline \multicolumn{6}{|c|}{ Área de domicílio } \\
\hline Urbana & 339 & 10,8 & $1,24(0,73-2,11)$ & 13,0 & $1,75(1,03-2,99)^{*}$ \\
\hline Rural & 205 & 9,6 & 1,00 & 8,4 & 1,00 \\
\hline \multicolumn{6}{|l|}{ NAF } \\
\hline Menos ativo & 234 & 9,1 & $0,90(0,54-1,51)$ & 11,8 & $1,15(0,71-1,87)$ \\
\hline Mais ativo & 307 & 11,1 & 1,00 & 11,1 & 1,00 \\
\hline
\end{tabular}

* $p<0,05$.

OR: odds ratio; IC95\%: intervalo de confiança; NAF: nível de atividade física. 
844 A. PELEGRINI et al.

\section{DISCUSSÃ O}

O presente estudo verificou o estado nutricional de adolescentes domiciliados na área urbana e na rural da região Sul do Brasil e analisou sua associação com fatores demográficos e com o NAF, vindo a contribuir como um estudo de base escolar preocupado em delimitar escolares quanto à área de domicílio (urbana e rural), tendo em vista as diferenças sociais e culturais existentes entre elas.

A prevalência de desnutrição encontrada nos escolares foi de $11,4 \%$. Prevalência superior de desnutrição foi encontrada na África ${ }^{20}$, Euro$\mathrm{pa}^{21}$ e América do Norte ${ }^{22}$. Em contrapartida, proporções inferiores foram verificadas na Ásia ${ }^{12}$. No Brasil, recente pesquisa conduzida em crianças menores de cinco anos revelou uma diminuição dos casos de desnutrição em $50 \%$ no período de $1996(13,5 \%)$ a $2006 / 7(6,8 \%)^{1}$. No mesmo estudo ${ }^{1}$, foi identificado que dois terços dessa redução poderiam ser atribuídos ao aumento da escolaridade materna, ao aumento do poder aquisitivo das famílias, à ampliação da assistência à saúde e à melhoria no saneamento básico.

A prevalência de excesso de peso foi de $11,2 \%$. Esses resultados são inferiores aos observados em pesquisas nacionais ${ }^{14} \mathrm{e}$ internacionais ${ }^{4,5}$. Por sua vez, esses achados são similares aos encontrados em adolescentes (14-18 anos) de João Pessoa (PB) $(10,0 \%)^{23}$ e em crianças (7-10 anos) de Porto Velho (RO) $(10,0 \%)^{24}$.

Prevalências mais elevadas de excesso de peso foram encontradas nos adolescentes de 10 a 13 anos. Esses resultados corroboram os evidenciados em adolescentes brasileiros ${ }^{25}$ : maiores prevalências de sobrepeso/obesidade foram verificadas naqueles com idade de 10 a 13 anos. Em contrapartida, divergem do estudo realizado em adolescentes de João Pessoa (PB) ${ }^{23}$, no qual a idade dos escolares não se associou à frequência de sobrepeso/obesidade em ambos os sexos. Em relação à área de domićilio, a prevalência mais elevada de excesso de peso foi encontrada nos adolescentes residentes na área urbana. Resul- tados semelhantes foram encontrados em pesquisa conduzida em adolescentes do Estado de Santa Catarina ${ }^{26}$.

Pesquisas sugerem que o comportamento sedentário esteja associado positivamente com o excesso de peso em ambos os sexos ${ }^{27}$. Todavia, os resultados do presente estudo demonstraram, para os rapazes, que os menos ativos fisicamente têm mais chance de apresentar desnutrição quando comparados àqueles mais ativos fisicamente. Leatherdale \& Wong ${ }^{28}$, ao investigar a relação de atividades sedentárias com o estado nutricional em mais de 25 mil crianças e adolescentes, encontraram que indivíduos do sexo masculino que gastavam mais tempo assistindo TV tiveram mais chance de apresentar desnutrição do que seus pares que gastavam menos tempo nessa atividade.

Os achados encontrados no presente estudo demonstraram, em ambos os sexos, associação entre excesso de peso e área de domicílio, tendo sido verificada maior chance de excesso de peso naqueles da área urbana. Esses resultados corroboram os observados em estudo internacional ${ }^{12}$ e nacional ${ }^{14}$, entretanto divergem dos relatados em adolescentes canadenses ${ }^{13}$. Uma possível explicação para a maior prevalência de excesso de peso nos escolares residentes na área urbana é o fácil acesso aos alimentos altamente calóricos (fast food) e/ou a diminuição dos níveis de atividade física. Já os adolescentes da área rural, muitas vezes, precisam se deslocar a pé para ter acesso ao transporte que os conduz até a escola, aumentando, dessa forma, o NAF e, consequentemente, diminuindo as chances de aumento do peso corporal. Além disso, no meio rural, culturalmente os filhos auxiliam os pais nas atividades laborais. Destaca-se que a região envolvida no estudo é caracterizada por minifúndios; assim, o trabalho braçal é necessário na execução das tarefas diárias, fazendo com que os adolescentes tenham um aumento no gasto energético diário.

Foi verificada, também, associação entre o excesso de peso corporal e o NAF nos rapazes. Os menos ativos fisicamente apresentaram 
maiores riscos de apresentar excesso de peso do que os mais ativos fisicamente. Esses resultados vão ao encontro da literatura ${ }^{29}$, que relaciona o excesso de peso com os baixos níveis de atividade física.

Nas moças, observou-se associação entre desnutrição e idade, o que revela que as adolescentes de 10 a 13 anos apresentaram maiores chances de desnutrição em relação às de 14 a 17 anos. Esse achado pode ser explicado pela influência da maturação sexual, pois, possivelmente, em faixa etária inferior - de 10 a 13 anos -, esse fenômeno ainda não ocorreu, ao contrário das adolescentes de 14 a 17 anos, nas quais as alterações na composição corporal são evidenciadas, aumentando-se, portanto, o risco de sobrepeso e obesidade ${ }^{30}$.

Entre as limitações do presente estudo destacam-se: 1) o fato de o estudo ser de base escolar, o que impede a generalização para jovens que não frequentam escola; 2) o desenho transversal, que impossibilita verificar a relação de causalidade entre as variáveis analisadas; 3 ) o fato de a maturação sexual não ter sido considerada, embora ela seja um fator capaz de alterar a avaliação do estado nutricional, especificamente na faixa etária investigada na presente casuística.

\section{O N C L U S Ã O}

Os resultados encontrados no presente estudo permitem concluir que a prevalência de desnutrição encontrada ainda é preocupante. Em relação ao excesso de peso, a prevalência encontrada se assemelha ao que tem sido observado nos estados da região Sul e Sudeste do Brasil. Os rapazes com baixo NAF apresentam maior exposição à desnutrição. Moças e rapazes da área urbana e rapazes com baixo NAF apresentaram maior exposição aos riscos decorrentes do excesso de peso corporal.

Para superar a situação da desnutrição, que ainda permeia o ambiente escolar, medidas eficientes e urgentes são exigidas para o combate à pobreza e à fome por meio da implementação de políticas de inclusão social. Além disso, atenção deve ser dada às condições inadequadas de saneamento básico, aos baixos níveis de educação e aos serviços de saúde deficientes. Além disso, medidas de intervenção, na infância e na adolescência, voltadas à prevenção, controle e tratamento do excesso de peso devem ser adotadas.

\section{COLABORADORES}

A. PELEGRINI e D.A.S. SILVA participaram de todo o processo de elaboração do artigo. E.L. PETROSKI participou da elaboração, revisão e análise crítica do artigo. M.F. GLANER participou da coleta dos dados, elaboração, revisão e análise crítica do artigo.

\section{REFERÊ N CIAS}

1. Monteiro CA, Benicio MHD, Konno SC, Silva ACF, Lima ALL, Conde WL. Causas do declínio da desnutrição infantil no Brasil, 1996-2007. Rev Saúde Pública. 2009; 43(1):35-43. doi: 10.1590/50034-89 102009000100005.

2. Coutinho JG, Gentil PC, Toral N. A desnutrição e obesidade no Brasil: o enfrentamento com base na agenda única da nutrição. Cad Saúde Pública. 2008; 24(Supl 2):S332-40. doi: 10.1590/S0102-31 $1 \times 2008001400018$.

3. Dietz WH. Childhood weight affects adult morbidity and mortality. J Nutr. 1998; 128(2):411S-4S.

4. Denney-Wilson E, Hardy LL, Dobbins T, Okely AD, Baur LA. Body mass index, waist circumference, and chronic disease risk factors in Australian adolescents. Arch Pediatr Adolesc Med. 2008; 162(6):566-73.

5. Del-Rio-Navarro BE, Velazquez-Monroy O, LaraEsqueda A, Violante-Ortiz R, Fanghanel G, PerezSanchez L, et al. Obesity and Metabolic Risks in Children. Arch Med Res. 2008; 39(2):215-21. doi: 10.1016/j.arcmed.2007.07.008.

6. Bibbins-Domingo K, Coxson P, Pletcher MJ, Lightwood J, Goldman L. Adolescent overweight and future adult coronary heart disease. N Engl J Med. 2007; 357(23):2371-9. doi: 10.1.1.126.2665.

7. Petry NM, Barry D, Pietrzak RH, Wagner JA. Overweight and obesity are associated with psychiatric disorders: results from the national epidemiologic survey on alcohol and related conditions. Psychosom Med. 2008; 70(3):288-97. doi: 10.1097/PSY.0b013e3181651651. 
8. Falkner NH, Neumark-Sztainer D, Story M, Jeffery RW, Beuhring T, Resnick MD. Social, educational, and psychological correlates of weight status in adolescents. Obes Res, 2001; 9(1):32-42. doi: 10.1038/oby.2001.5

9. World Health Organization. Preventing chronic disease: a vital investment. Geneva: WHO; 2005 [cited 2008 Jun 17]. Available from: <www.who. int/chp/chronic_disease_report>.

10. Rennie KL, Johnson L, Jebb SA. Behavioural determinants of obesity. Best Pract Res Clin Endocrinol Metab. 2005; 19(3):343-58. doi: 10.10 16/j.beem.2005.04.003.

11. Pietiläinen KH, Kaprio J, Borg P, Plasqui P, YkiJärvinen $H$, Kujala UM, et al. Physical inactivity and obesity: a vicious circle. Obesity. 2008; 16(2): 409-14.

12. Tang HK, Dibley MJ, Sibbritt D, Tran HM. Gender and socio-economic differences in BMI of secondary high school students in Ho Chi Minh city. Asia Pac J Clin Nutr. 2007; 16(1):74-83.

13. Bruner MW, Lawson J, Pickett W, Boyce W, Janssen I. Rural Canadian adolescents are more likely to be obese compared with urban adolescents. Int J Pediatr Obes. 2008; 3(4):205-11. doi: 10.1080/174 77160802158477.

14. Instituto Brasileiro de Geografia e Estatística. Antropometria e análise do estado nutricional de crianças e adolescentes no Brasil. Pesquisa de Orçamentos Familiares, 2002-2003. Brasília: IBGE; 2006.

15. Gordon CC, Chumlea WC, Roche AF. Stature, recumbent length, and weight. In: Lohman TG, Roche AF, Martorell R, editors. Anthropometric standardization reference manual. Abridged edition. Champaign: Human Kinetics Books; 1991. p.3-8.

16. Cole TJ, Flegal KM, Nicholls D, Jackson AA. Body mass index cut offs to define thinness in children and adolescents: international survey. BMJ. 2007; 335(7612): 194. doi: 10.1136/bmj.39238.3994 44.55.

17. Cole TJ, Bellizzi MC, Flegal KM, Dietz WH. Establishing a standard definition for child overweight and obesity worldwide: international survey. BMJ. 2000; 320(7244):1240-3. doi: 10.11 36/bmj.320.7244.1240.

18. Pate RR. Recent statements and initiatives on physical activity and health. Quest. 1995; 47(3): 304-10.

19. Nahas MV. Atividade física, saúde e qualidade de vida: conceitos e sugestões para um estilo de vida ativo. $2^{a}$ ed. Londrina: Midiograf; 2001.
20. Aounallah-Skhiri H, Romdhane HB, Traissac P, Eymard-Duvernay S, Delpeuch F, Achour N, et al. Nutritional status of Tunisian adolescents: associated gender, environmental and socio-economic factors. Public Health Nutr. 2008; 11(12):1306-17. doi: 10.10 17/S1368980008002693.

21. Oblacinska A, Tabak I, Jodkowska M. Demographic and regional determinants of underweight in Polish teenagers. Przegl Epidemiol. 2007; 61(4):785-93.

22. Wang Y, Monteiro C, Popkin BM. Trends of obesity and underweight in older children and adolescents in the United States, Brazil, China, and Russia. Am J Clin Nutr. 2002; 75(6):971-7.

23. Farias Júnior JC, Silva KS. Sobrepeso/obesidade em adolescentes escolares da Cidade de João Pessoa - PB: prevalência e Associação com Fatores Demo-gráficos e Socioeconômicos. Rev Bras Med Esporte. 2008; 14(2):104-8. doi: 10.1590/\$1517-86 922008000200004.

24. Farias ES, Guerra-Júnior G, Petroski EL. Estado nutricional de escolares em Porto Velho, Rondônia. Rev Nutr. 2008; 21(4):401-9. doi: 10.1590/\$1415-52 732008000400004.

25. Pelegrini A, Petroski EL, Coqueiro RS, Gaya ACA. Overweight and obesity in Brazilian schoolchildren aged 10 to 15 years: data from a Brazilian sports project. Arch Latinoam Nutr. 2008; 58(4):343-9.

26. Silva KS, Nahas MV, Hoefelmann LP, Lopes AS, Oliveira ES. Associações entre atividade física, índice de massa corporal e comportamentos sedentários em adolescentes. Rev Bras Epidemiol. 2008; 11(1): 159-68. doi: 10.1590/S1415-790X2008000100 015.

27. Hancox RJ, Milne BJ, Poulton R. Association between child and adolescent television viewing and adult health: a longitudinal birth cohort study. Lancet. 2004; 364(9430):257-62. doi: 10.1016/S0 140-6736(04)16675-0.

28. Leatherdale ST, Wong SL. Modifiable characteristics associated with sedentary behaviours among youth. Int J Pediatr Obes. 2008; 3(2):93-101. doi: 10.1080/17477160701830879.

29. Hassink SG, Zapalla F, Falini L, Datto G. Exercise and the obese child. Prog Pediatr Cardiol. 2008; 25(2):153-7. doi: 10.1016/j.ppedcard.2008.06.001.

30. Oliveira CS, Veiga GV. Estado nutricional e maturação sexual de adolescentes de uma escola pública e de uma escola privada do Município do Rio de Janeiro. Rev Nutr. 2005; 18(2):183-91. doi: 10.15 90/S1415-52732005000200002.

Recebido em: 24/10/2008

Versão final reapresentada em: 12/1/2010 Aprovado em: 31/5/2010 